\title{
Instrumentierung und Fusion nach thorakalem und lumbalem Trauma
}

\author{
Oliver Gonschorek, Stefan Hauck, Volker Bühren
}

\section{Zusammenfassung}

Die operative Versorgung von thorakalen und lumbalen Wirbelsäulenverletzungen folgt einem differenzierten Algorithmus, der biologisches Alter und Knochenqualität des Patienten sowie Instabilitätsgrad und Lokalisation der Verletzung berücksichtigt. Wesentlich hierbei ist eine subtile Diagnostik unter Einsatz schnittbildgebender Verfahren und eine genaue Klassifikation vorzugsweise nach AO. Zahlenmäßig am häufigsten kommen Wirbelkörperfrakturen im thorakolumbalen Übergang vor. Aufgrund der Kyphosierung und der entsprechend hohen Belastung der ventralen Säule in diesem Bereich ist die ventrale Instrumentierung hier einerseits besonders wichtig, andererseits mittlerweile dank des Einsatzes minimalinvasiver thorakoskopischer Techniken, aber auch durch eine niedrige additive Zugangsmorbidität ausgezeichnet. Die Kombination dieser minimalinvasiven, endoskopisch gestützten Technik mit modernen Implantaten wie expandierbare Cages und winkelstabile Plattensysteme ermöglichen heute die konsequente belastungsstabile Rekonstruktion der vorderen Säule bei gleichzeitig deutlich reduzierter Morbidität. Die dorsale In- strumentierung dient wesentlich der Reposition bei Kyphose-Fehlstellung nach Berstungsbrüchen und der Stabilisierung bei B- und C-Verletzungen. Im oberen thorakalen Bereich ist die Indikation zur operativen Therapie seltener und kann oft auf die dorsale Instrumentierung beschränkt bleiben. Die ventrale Rekonstruktion ist aber auch hier bis etwa T5 in thorakoskopischer Technik gut möglich. Rein prinzipiell ist bei A-Verletzungen das rein ventrale Vorgehen ausreichend, eine dorsale Instabilität muss dann aber sicher ausgeschlossen werden. Bei älteren Patienten ist zu entscheiden, ob eine osteoporotische Fraktur nach Bagatelltrauma vorliegt oder eine traumatische Fraktur bei osteoporotischem Knochen. Während bei ersterem eine Kyphoplastie alleine ausreichend sein kann, muss bei letzterer Konstellation eine augmentierte Versorgung mit Fixateur interne in Erwägung gezogen werden.

\section{Instrumentation and Fusion after Thoracic and Lumbar Spine Injuries}

The operative treatment of thoracic and lumbar spine fractures follows a differentiated algorithm. Biological age and bone quality of the patient as well as grade of instability and localisation of the injury have to be recognised. X-ray images and especially CT scans allow one to classify the injury correctly according to the AO. Most of the fractures are focused on the thoracolumbar region, where the kyphotic spine leads to a high axial load on the anterior column. Therefore, anterior instrumentation plays a major role in this region. New minimally invasive endoscopic assisted techniques and new implants such as expandable cages and anglestable plates allow one to follow this algorithm with significantly reduced additive morbidity for the patient. Posterior instrumentation allows for sufficient reduction of post-trauma kyphosis in A fractures if performed in the acute phase and can also be performed in $B$ and $C$ type fractures. Fractures of the upper and middle thoracic spine do not need anterior reconstruction so frequently. Such a reconstruction is, however, possible up to T5, then approaching from the right side. Isolated anterior reconstruction may be sufficient in $A$ type fractures if posterior instability is excluded. Elderly patients often suffer from osteoporotic fractures and need kyphoplasty. In cases of high energy trauma they also need posterior instrumentation which may be performed under augmentation with cement.

\section{Einleitung}

Zunehmende Freizeitaktivitäten im Hochrasanzbereich, aber auch weiterreichende operative Techniken unter Einbeziehung minimalinvasiver Methoden haben $\mathrm{zu}$ einem stetigen Bedeutungszuwachs von Wirbelsäulenverletzungen und deren operativer Therapie im Spekt-

OP-JOURNAL 2009; 25: 12-18

(c) Georg Thieme Verlag KG Stuttgart · New York DOI 10.1055/s-0029-1185514 rum von unfallchirurgischen Kliniken geführt. Zusätzlich führen gesteigerte Ansprüche an die uneingeschränkte Mobilität auch im hohen Alter zu einem vermehrten Anteil von älteren Patienten [3, 7].

Im Rahmen der Wirbelsäulenverletzungen nimmt die thorakolumbale Region eine hervorragende Stellung ein. 70$80 \%$ der relevanten Verletzungen betreffen Brust- und Lendenwirbelsäule, davon wiederum etwa $70 \%$ den thorakolumbalen Übergang. Die erhöhte Anfälligkeit dieser Region ist bedingt durch den Übergang von der Kyphose der Brustwirbelsäule (BWS), die zudem eine zusätzliche Stabilisierung durch den Brustkorb erfährt, zur Lordose der freistehenden Lendenwirbelsäule (LWS).

Die Indikation zur Operation basiert auf 3 Pfeilern: Neurologie, Instabilität, Fehlstellung. Dies impliziert auch die notwendige Notfalldiagnostik. An die Übersichts-Röntgenaufnahmen in 2 Ebenen sind bei Verdacht auf eine Wirbelsäulenverletzung eine Computertomografie 
(CT) mit Rekonstruktionen sowie eine differenzierte neurologische Untersuchung anzuschließen. Eine einheitliche Klassifikation ist aufgrund der komplexen Strukturen der Wirbelsäule unabdingbar. Sie erleichtert den behandelnden Ärzten die Kommunikation, erlaubt Rückschlüsse auf den Behandlungserfolg verschiedener operativer Strategien und ermöglicht den Vergleich verschiedener Patientenkollektive $[3,6]$.

Die Behandlungskonzepte zur Versorgung thorakolumbaler Frakturen wird nach wie vor auf internationaler Ebene kontrovers diskutiert. Im deutschen Sprachraum hat sich insbesondere die AG Wirbelsäule der DGU mit 2 groß angelegten Multicenterstudien mit diesem Thema beschäftigt und konnte die besondere Bedeutung der ventralen Säule herausarbeiten. Biomechanische Untersuchungen unterstützen diese Beobachtungen und neue minimalinvasive Techniken erlauben das Umsetzen im klinischen Alltag bei deutlich reduzierter Zugangsmorbidität [1,9,11,13-15].

Der hohe logistische Aufwand bindet die differenzierte moderne Versorgungsstrategie an das Zentrum. Nur hier sind die Voraussetzungen für die jederzeit auch notfallmäßig - mögliche Komplexversorgung einschließlich anhängiger Intensivbetreuung und ggfs. erforderlichen Komplikations- und Korrektureingriffe gegeben [6].

\section{Diagnostik}

In der Notfalldiagnostik bleibt das konventionelle Röntgen die erste Wahl. Bei Frakturverdacht ist die entsprechende Region durch eine Computertomografie (CT) mit Rekonstruktionen - zunehmend auch im 3D-Modus - weiter abzuklären. In etwa $30 \%$ der Polytraumatisierten liegt eine Wirbelsäulenverletzung vor, hier ist standardmäßig primär ein Polytrauma-CT diagnostische Grundlage. Das Kernspintomogramm (MRT) bleibt speziellen Fragestellungen vorbehalten, so bei Neurologie ohne Hinweis einer knöchernen Läsion oder Instabilität, und bei abklärungsbedürftigen Befunden an der Bandscheibe. Weiterhin kann das MRT zum Ausschluss einer dorsalen Bandinstabilität dienen, wenn ein isoliert ventrales operatives Vorgehen geplant wird.

Das CT ist die Grundlage für Klassifikation und Therapieplanung bei Wirbelsäulenverletzungen.

\section{Klassifikation}

Eine brauchbare Klassifikation erlaubt die Identifizierung jeder Verletzung durch möglichst einfache Algorithmen basierend auf klinischen und radiologischen Charakteristika. Sie soll reproduzierbar Hinweise zu Verletzungsschwere und Therapiewahl geben und so Grundlage für klinische Studien sein.

Die heute gebräuchliche Klassifikation für Wirbelsäulenverletzungen im deutschsprachigen Raum orientiert sich an der Einteilung der Arbeitsgemeinschaft für Osteosynthesefragen (AO) und wurde von der Arbeitsgruppe Magerl, Harms, Gertzbein, Aebi und Nazarian ausgearbeitet. Die Systematik ordnet mit zunehmender Instabilität von $\mathrm{A}$ nach $\mathrm{C}$ und orientiert sich an dem Zweisäulenmodell nach Whitesides. Isolierte Queroder Dornfortsatzfrakturen bleiben unberücksichtigt [12].

A-Frakturen betreffen den Wirbelkörper und damit die ventrale, in der Regel auf Kompression belastete, Säule. B- und CVerletzungen betreffen beide Säulen, wobei Typ B durch einen Flexions-/Distraktionsmechanismus und Typ $C$ durch rotatorische Instabilität gekennzeichnet sind.

Die häufigste Form der A-Verletzungen ist durch eine intakte Hinterkante gekennzeichnet und wird in aller Regel konservativ behandelt (A1). Die schwerwiegendste Form des Spaltbruchs (A2) ist der Kneifzangenbruch (A2.3); er führt in der Regel zu einer Destruktion beider benachbarter Bandscheiben und ist dorsoventral bisegmental operativ anzugehen.

Der Berstungsbruch ist der häufigste zur operativen Therapie führende Bruchtypus überhaupt; man unterscheidet inkompletten (A3.1) und kompletten (A3.3) Berstungsbruch vom Berstungsspaltbruch (A3.2). Die beiden letzten sind bisegmental, ersterer bei guter Knochenqualität auch monosegmental von ventral zu versorgen.

Eine Sonderform stellt die osteoporotische Fraktur des älteren Patienten dar, in der Regel liegt sie als A1.3 vor.

Bei B-Verletzungen liegt der biomechanische Pathomechanismus in einer Kombination aus Flexion und Distraktion (B1, B2) bzw. in einer Hyperextension (B3). B1-Verletzungen betreffen dorsal die ligamentären, B2-Verletzungen die knö- chernen Strukturen. Als Sonderform sei die Chance-Fraktur (B2.1) erwähnt, die dorsale knöcherne Verletzung läuft über die Bögen nach ventral knöchern ohne größere Destruktion im Wirbelkörper aus.

Rotatorische Dislokations- und Instabilitätskomponenten charakterisieren die komplexeste Form der Wirbelsäulenverletzungen, den C-Typ. Regelhaft liegen Hochrasanztraumata oder der Sturz aus hoher Höhe vor, entsprechend hoch ist der Anteil von Begleitverletzungen, Polytrauma und Querschnittsläsionen. Entsprechend der hochgradigen Instabilität werden diese Frakturformen praktisch ausnahmslos operativ behandelt.

\section{Dorsale Instrumentierung}

Die dorsale Instrumentierung mit winkelstabilem Fixateur interne stellt nach wie vor die Routinetechnik zur akuten operativen Versorgung einer instabilen Verletzung der thorakalen und lumbalen Wirbelsäule dar. Dabei sind Instabilitäten als dringliche, neurologische Ausfallerscheinungen als Notfallindikationen zu betrachten. Neben der Korrektur der frakturbedingten kyphotischen Fehlstellung gelingt regelhaft über Ligamentotaxis auch die indirekte Dekompression bei Spinalkanaleinengung $[6,8]$.

Aufgrund biomechanischer Überlegungen sind die rein dorsalen Instrumentierungen gegenüber der Rekonstruktion der ventralen Säule deutlich in den Hintergrund getreten. B-Frakturen mit knöcherner Komponente (Chance-Fraktur) oder Bandscheibenbeteiligung mit guter Heilungstendenz (bei sehr jungen Patienten) stellen sicher noch gute Indikationen für das rein dorsale Vorgehen dar. Die Gefahr des sekundären Repositionsverlusts ist gerade bei Berstungsbrüchen hoch, die eigentliche Pathologie der destruierten Bandscheibe kann durch die rein dorsale Instrumentierung nicht kausal angegangen werden.

Bei neurologischer Symptomatik und entsprechender Spinalkanalverlegung ist eine Laminektomie im Rahmen der Akutversorgung zu diskutieren. Sie stellt zwar eine weitere Destabilisierung dar, kann aber rasch das Myelom dekomprimieren. Diese Ausnahmeindikation ist am ehesten in der oberen bis mittleren BWS zu sehen, wo der Spinalkanal sehr eng um das Myelom anliegt. Intraoperativ können sonografische Kontrollen die Raumverhältnisse nachweisen, Myelo- 
grafien sind meist nur eingeschränkt aussagefähig. Hier ist künftig von intraoperativ einsetzbaren 3D-Bildwandlersystemen ein wesentlicher Fortschritt zu erwarten.

Der Einsatz einer Querstrebe kann bei hochinstabilen Verletzungen oder bei additiver Destabilisierung durch eine Laminektomie die primäre Stabilität erhöhen. In gleichem Sinne kann es erforderlich sein, eine multisegmentale dorsale Versorgung vorzunehmen, so z. B. bei Tumoren oder Metastasen, bei osteoporotischen Verhältnissen oder bei hoch thorakalen Versorgungen, bei denen relativ dünne Pedikelschrauben eingesetzt werden.

Zunehmender Trend ist die Entwicklung minimalinvasiv einsetzbarer dorsaler Instrumentierungen. Problematisch ist hier allerdings weiterhin die limitierte Repositionsmöglichkeit dieser Systeme, wobei mittelfristig Fortschritte zu erwarten sind.

Bei osteoporotischem Knochen kommen zunehmend zementaugmentierte Instrumentierungen zum Einsatz, die dann häufig auch mit der Kyphoplastie des betroffenen Wirbelkörpers kombiniert werden.

Bei rascher Versorgung und konsequenter Durchführung kann die dorsale Instrumentierung eine gute Reposition auch von dislozierten Hinterkantenfragmenten erzielen.

\section{Ventrale Spondylodese}

Aus der biomechanischen Überlegung der druckbelasteten A-Säule und der Erkenntnis der Bandscheibenpathologie bei Berstungsbrüchen heraus hat die Rekonstruktion der ventralen Säule einen wesentlichen Bedeutungszuwachs erfahren. Mit dazu beigetragen hat die Entwicklung minimalinvasiver, endoskopisch gestützter Techniken und der damit deutlich gesenkten Zugangsmorbidität sowie expandierbarer Wirbelkörperersatzsysteme und winkelstabiler Implantate. Die Repositionsverluste nach alleiniger dorsaler Instrumentierung können so signifikant reduziert werden $[1-6,8]$.

\section{Dorsoventrale Instrumentierung}

Berstungsbrüche (A3) sollten dringlich dorsal instrumentiert werden. Die Reposition, insbesondere auch des Hinterkan-
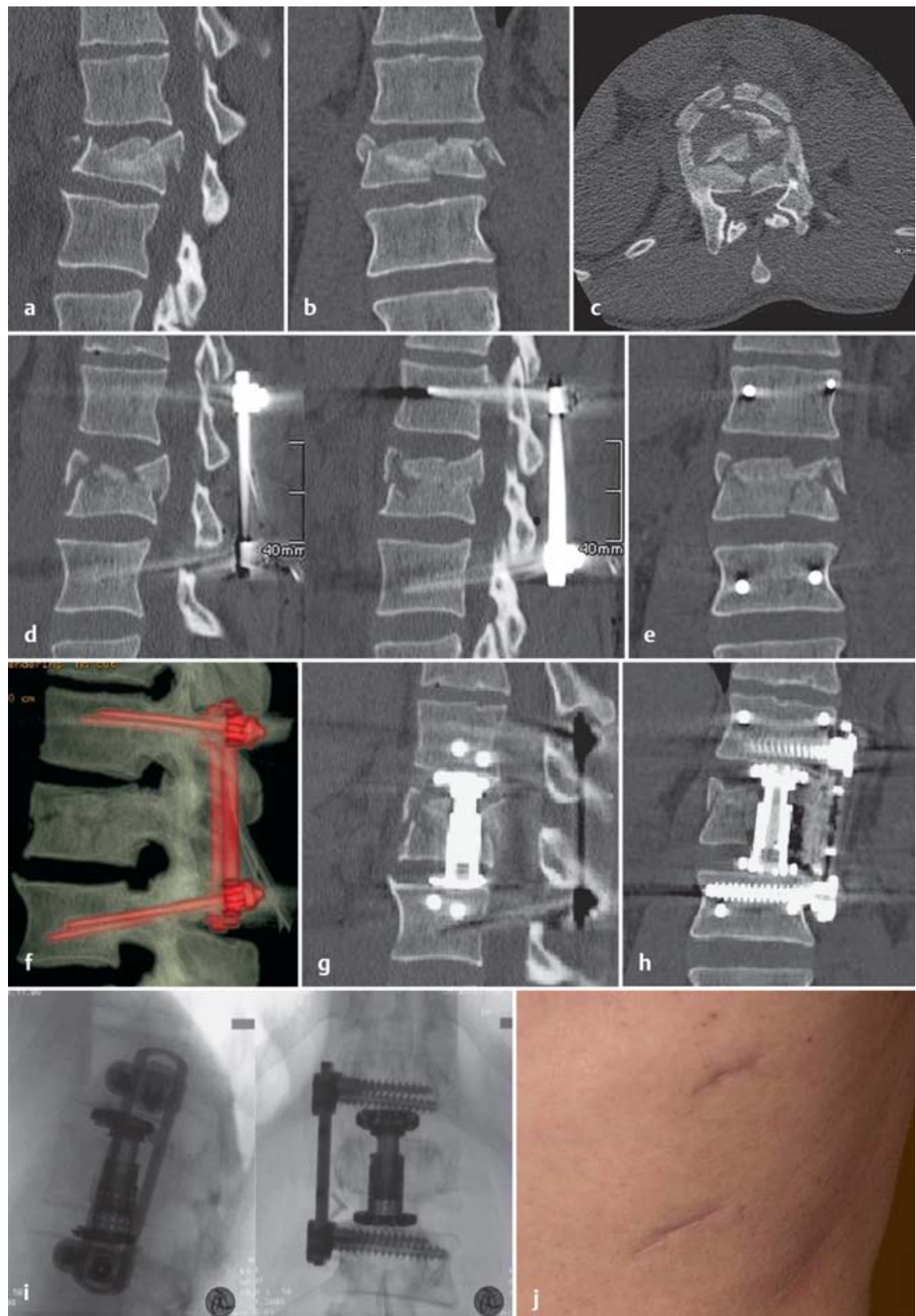

Abb. 1 a bis j Berstungsspaltbruch A3.2 des LWK1 bei einem 48-jährigen Mann. CT bei Aufnahme: sagittal (a) zeigt die Berstung im kranialen Anteil und die kyphotische Fehlstellung; frontal (b) erkennt man gut die Spaltkomponente; die axiale Schnittführung (c) verdeutlicht die Berstungskomponente und die Spinalkanaleinengung. Das postop. CT zeigt sagittal (d) die gute Reposition, das dorsale Kantenfragment ist dennoch nicht komplett reponiert; frontal (e) erkennt man eine gute Aufrichtung. Die 3D-Rekonstruktion (f) verdeutlicht das OP-Ergebnis. Nach thorakoskopisch gestützter ventraler bisegmentaler Spondylodese steht der Synex2-Cage korrekt ( $\mathbf{g}$, sagittaler Schnitt); in der frontalen Ebene erkennt man die additive winkelstabile Platte (h). Verlaufskontrolle radiologisch (i) und klinisch mit problemloser Wundheilung und kleinen Narben im ventralen Zugangsgebiet (j).

tenfragments, gelingt akut am besten. In 2. Sitzung kann dann elektiv die Abstützung der ventralen Säule in der Regel über einen minimalinvasiven vorderen Zugang endoskopisch gestützt erfolgen. Bei inkompletten Berstungsbrüchen vom Typ A3.1 kann eine monosegmentale ventrale Spondylodese ausreichen, das 2. Bewegungssegment sollte dann durch eine Materialentfernung des Fixateur interne nach etwa einem Jahr freigegeben werden. Berstungsspaltbrüche (A3.2) und komplette Berstungsbrüche (A3.3) erfordern ebenso wie Kneifzangenbrüche (A2.3) regelhaft eine bisegmentale ventrale Spondylodese (Abb.1). In diesen Fällen kann der Fixateur interne prinzipiell auf Dauer belassen werden, 


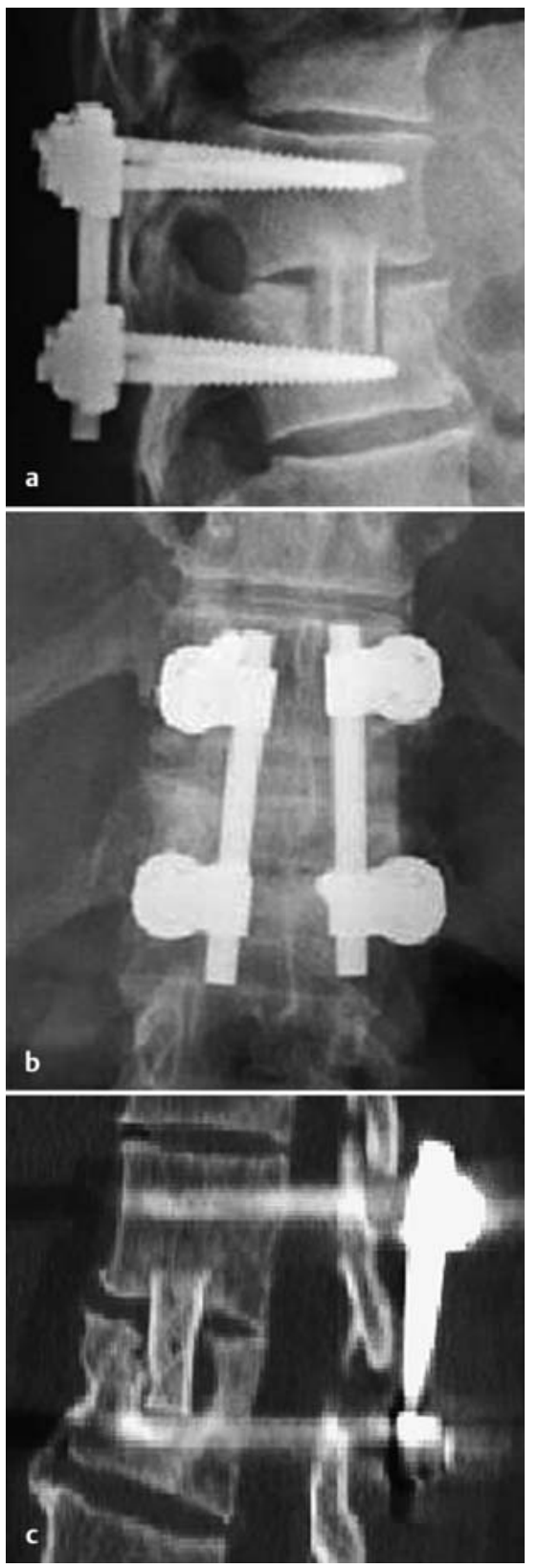

Abb. 2a bis c Nach dorsoventral monosegmentaler Versorgung zeigt sich im Röntgen eine korrekte Stellung des Spanes (a, b). Erst im CT wird die Problematik der Spaneinheilung deutlich (c).

da es sich um eine Rahmenkonstruktion handelt. Ventrale Implantate werden prinzipiell belassen $[5,6,8]$.

\section{Isoliert ventrale Spondylodese}

Prinzipiell kann bei A-Verletzungen ein rein ventrales Vorgehen zum Einsatz kommen. Dann muss allerdings eine dorsale Pathologie im Sinne einer B-Verletzung sicher ausgeschlossen sein, was selbst unter dem Einsatz des MRT nicht

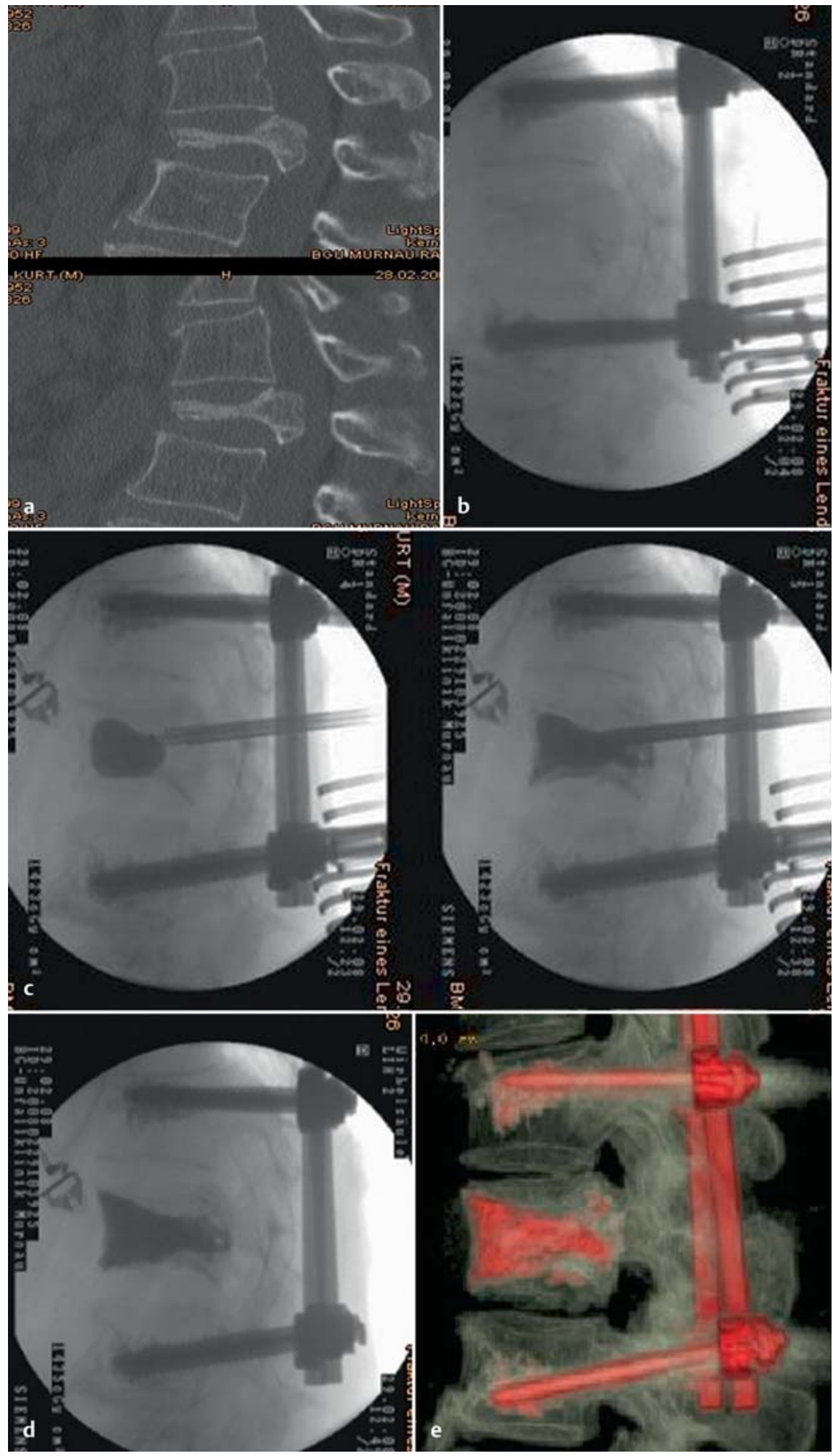

Abb. 3 a bis e Vertebra plana bei einem osteoporotischen Wirbelkörperbruch (a). Dorsale Instrumentierung zementaugmentiert (b) erzielt zusammen mit einer Ballonkyphoplastie (c) eine vollständige Aufrichtung des Wirbelkörpers (d). Postoperative 3D-CT-Darstellung des Ergebnisses (e).

immer sicher möglich ist. Limitierend ist weiterhin die eingeschränkte Repositionsmöglichkeit durch die ventralen Systeme.
Insofern ist diese Vorgehensweise Frakturen mit eher geringer Kyphose beim jungen Patienten mit guter Knochenqualität vorbehalten $[6,8]$. 


\section{Mono-/bisegmentale ventrale Spondylodese}

Die bisegmentale ventrale Spondylodese mit expandierbarem Wirbelkörperersatz (Cage) wird von den Autoren als Regelversorgung zur Rekonstruktion der ventralen Säule angesehen. Die additive winkelstabile Platte erhöht die Stabilität, ist allerdings nicht in allen Fällen apodiktisch zu fordern. Der Einsatz von Knochenspänen bei der bisegmentalen Versorgung wurde aufgrund der Entnahmemorbidität und der Einheilungsproblematik im eigenen Patientengut weitestgehend verlassen (Abb. 2).

Bei inkompletten Berstungsbrüchen kann durch die monosegmentale Versorgung ein Bewegungssegment erhalten werden. Voraussetzung hierfür sind allerdings ein ausreichender Knochenstock des betroffenen Wirbelkörpers und eine gute Knochenqualität. Der Einsatz von Knochenspänen konkurriert bei dieser Indikation vor allem mit neu entwickelten erweiterten Bandscheibenersatzsystemen, die durch ihre wabenartige Struktur und spezielle Materialbeschaffenheit den knöchernen Durchbau begünstigen sollen bei gleichzeitiger hoher Primärstabilität. Die additive winkelstabile Platte ist bei der monosegmentalen Versorgung als unverzichtbar anzusehen.

\section{Vertebroplastie/Kyphoplastie}

Eine Sonderform stellt die osteoporotische Fraktur des älteren Menschen dar. Bei der Indikationsstellung zur Operation müssen immer auch die Begleiterkrankungen und damit das OP- und Narkoserisko bedacht werden. Häufig liegen A1.3-Frakturen vor, die prinzipiell konservativ behandelt werden können. Im Vordergrund der Indikation zur Zementaugmentierung steht somit auch die erhebliche persistierende Schmerzsymptomatik. Während bei der Kyphoplastie durch Einsatz eines auffüllbaren Ballons oder ähnlicher Mechanismen eine Höhle geschaffen wird, sieht die Vertebroplastie das Einbringen des Zements in den unaufbereiteten Wirbelkörper vor. Vor- und Nachteile der jeweiligen Technik werden in der Literatur kontrovers diskutiert.

Immer häufiger findet man aber auch beim älteren Patienten Wirbelsäulenverletzungen nach adäquatem Trauma. Die alleinige Zementaugmentierung ist dann nicht mehr ausreichend und muss durch
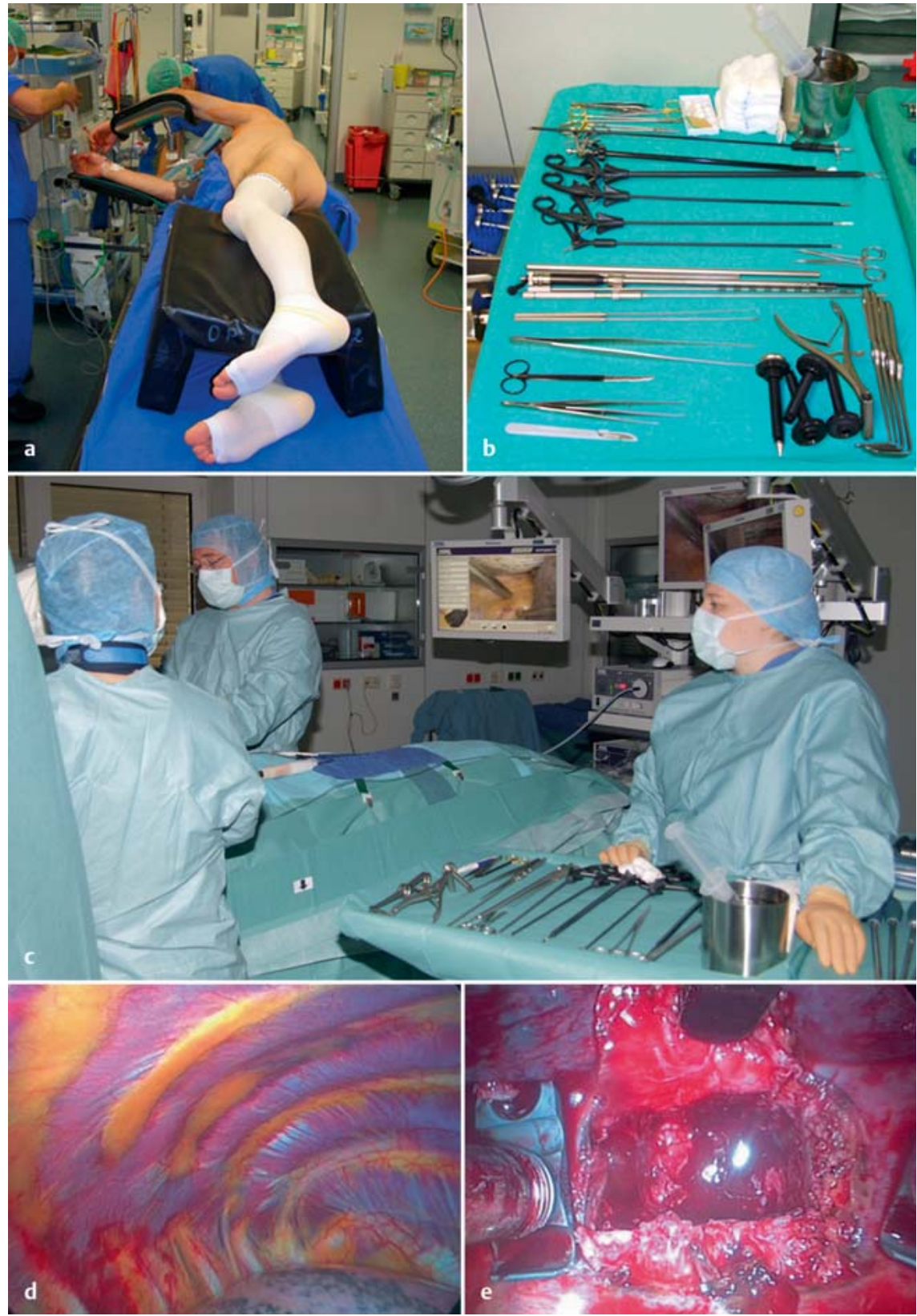

Abb. 4a bis e Die minimalinvasive ventrale Technik, endoskopisch gestützt, erfordert einen hohen technischen Aufwand. Der Patient wird in einem speziellen Vakuumkissen auf die Seite gelagert (a), spezielles Instrumentarium ist unabdingbar (b). Sowohl Operateur als auch Assistenten und OP-Schwestern/Pfleger können die gesamte OP am Bildschirm verfolgen (c). Exzellente Übersicht intrathorakal (d). Beispielhafte Situs-Darstellung nach subtotaler Korporektomie (e).

Kombination mit der dorsalen Instrumentierung, die dann ebenfalls in augmentierter Form vorzunehmen ist, ergänzt werden (Abb. 3). Im eigenen Patientenkollektiv nimmt auch die dorsoventrale Komplettversorgung unter Einsatz des expandierbaren Cages beim Älteren an Bedeutung zu. Auf der einen Seite ist hier die schonende minimalinvasive Technik vorteilhaft, andererseits nimmt der Anspruch auch der älteren Patienten an die Versorgung zu. Entspre- chend steigt auch der Anteil an Revisionsoperationen beim nicht ganz jungen Patienten gerade nach fehlgeschlagenen Kyphoplastie-Versorgungen, worüber weiter unten noch berichtet wird.

Zementaugmentierte Techniken ermöglichen eine adäquate Therapie von osteoporotischen Frakturen bei älteren Patienten, wobei die Schmerzreduktion im Vordergrund steht. 

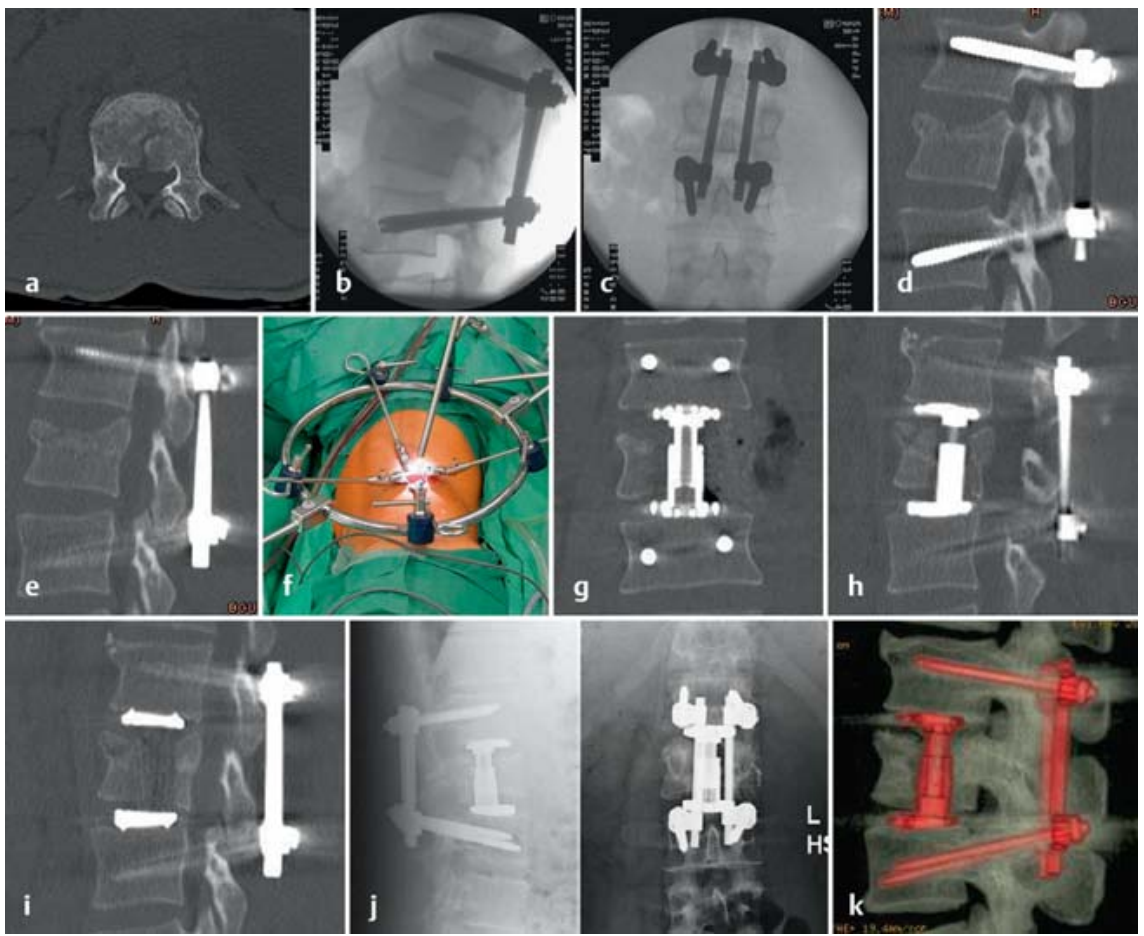

Abb. $\mathbf{5}$ a bis $\mathbf{k}$ Inkompletter Berstungsbruch A3.1 des LWK2 bei einem 50-jährigen Mann. Im axialen CT-Schnitt zeigt sich die Berstungskomponente mit fast $50 \%$ iger Spinalkanaleinengung (a). Durch die akute dorsale Instrumentierung mit USS-Schanz-System kann eine komplette Reposition erzielt werden (intraop. Kontrolle, b, c). Das postoperative CT weist die korrekte Reposition, insbesondere des Hinterkantenfragments nach (d, e). Es schließt sich der ventrale Eingriff an, endoskopisch gestützt, über eine Minilumbotomie, unter Einsatz des Synframe (f). Das postoperative CT zeigt die korrekte Platzierung des Synex2-Cages sagittal $(\mathbf{g})$ und frontal (h), insbesondere die ausgezeichnete Anpassung der anatomisch geformten Endplatten des Synex2Cages an Grund- und Deckplatte der angrenzenden Wirbelkörper (i). Das postoperative Ergebnis wird in 2 Ebenen dokumentiert (j) und im 3D-CT nochmals detailliert dargestellt (k).

\section{Minimalinvasive ventrale OP-Technik}

Grundvoraussetzung für die thorakoskopische Technik ist die Einlungenbeatmung unter Einsatz eines Doppellumentubus durch die Anästhesie. Der Patient wird in Seitlage gebracht, wobei vorteilhaft ein Vakuumbett als Schale fungiert. Wichtig ist die Auslagerung der Arme ohne Zug an den Schultern, um Plexusirritationen zu vermeiden (Abb. 4). Unter BV-Kontrolle wird das Zugangsgebiet angezeichnet. Regelhaft werden 3 Zugänge von jeweils etwa $2 \mathrm{~cm}$ Durchmesser und ein etwas größerer Zugang, über den Cage und Platte eingebracht werden, benötigt. Beim ersten Zugang ist besondere Vorsicht geboten, alle weiteren werden unter strenger thorakoskopischer Kontrolle gesetzt. Der Doppellumentubus ermöglicht das kontrollierte Kollabieren der Lunge auf der Zugangsseite (ab Th8 links, weiter kranial ggfs. rechts). Mit einem ausfahrbaren Spreizer können Lunge oder Zwerchfell bei Bedarf beiseite gehalten bzw. angespannt werden. Auf Höhe L1/L2 ist regelhaft ein Zwerchfellsplit erforderlich, hier hat sich der Ein- satz des Ultraschallmessers bewährt [1, 2,9-11].

Unter BV-Kontrolle werden die zu instrumentierenden Wirbelkörper aufgesucht und kranial und kaudal wird je eine Schraube gesetzt. Dies erleichtert bei der weiteren Präparation die Orientierung. Die Segmentgefäße sind bei Bedarf aufzusuchen und nach Setzen von Clips zu durchtrennen. Zur Korporektomie (partiell oder subtotal, je nachdem ob eine mono- oder bisegmentale Spondylodese vorgesehen ist) wird zunächst die Bandscheibe scharf am gesunden Wirbelkörper abgetrennt, der betroffene Wirbelkörper wird mit dem Meißel präpariert. Wirbelkörper- und Bandscheibenreste werden unter abwechselnder Verwendung von Rangeur, Stanze, Curette und Meißel entfernt. Backtable erfolgt die Separation von verwendbaren Knochenanteilen, die in einer Knochenmühle zur Spongiosaplastik aufbereitet werden. Nach Einbringen und Aufspreizen des expandierbaren Cages (bisegmental) oder Einbolzen des Knochenspans bzw. erweiterten Bandscheibenersatzes (mo- nosegmental) wird die seitliche winkelstabile Plattenosteosynthese komplettiert. Die Zwerchfelllücke wird verschlossen, eine Thoraxdrainage wird eingelegt, die Lunge gebläht, jeweils unter Sicht des Thorakoskops.

Bei Verletzungen der oberen Brustwirbelsäule kann die Lage von Herz und Aorta den Zugang von rechts erfordern. Die kleineren Wirbelkörper und die sehr enge Beziehung des Myeloms zum knöchernen Spinalkanals machen die operative Technik in dieser Höhe noch anspruchsvoller.

Unter Einsatz des Zwerchfellsplits ist der Zugang bis zu L2 regelhaft in thorakoskopischer Technik möglich. Weiter kaudal muss retroperitoneal zugegangen werden. Auch dies ist minimalinvasiv im Sinne einer Minilumbotomie durchführbar, vorteilhaft ist hier der Einsatz von Retraktorsystemen, das Endoskop kommt auch hier zum Einsatz, um Instrumenteur und Assistenz über den Bildschirm am Eingriff teilhaben zu lassen (Abb.5).

Durch den Einsatz minimalinvasiver Techniken und moderner Implantate wie z.B. die expandierbaren Cages hat die Rekonstruktion der ventralen Säule erheblich an Bedeutung gewonnen.

\section{Korrektureingriffe}

Kyphotische Fehlstellungen, unversorgt gebliebene destruierte Bandscheiben und in zunehmendem Maße fehlgeschlagene Kyphoplastien führen bei entsprechender Beschwerdesymptomatik zur Indikation der Revisions- bzw. Korrekturoperation. Hierbei handelt es sich um sehr aufwendige Techniken. Neben der kombiniert dorsoventralen Vorgehensweise ist vor allem das ventrale Release zu erwähnen, das in unmittelbarer Nachbarschaft der Aorta vorzunehmen ist. Entsprechend kann hier die Navigation besonders vorteilhaft zum Einsatz kommen. Die Korrektureingriffe starten in Bauchlage zur Implantation eines Fixateur interne. Eine signifikante Lordosierung kann allerdings regelhaft erst nach ventraler Präparation im Sinne eines ventralen Release erzielt werden. Hierzu muss dann in Seitlage die dorsale Instrumentierung weiter zugänglich und manipulierbar sein. Ggfs. kann der dorsale Part in Form eines transkutanen temporär eingesetzten Fixateur externe beschränkt bleiben. 


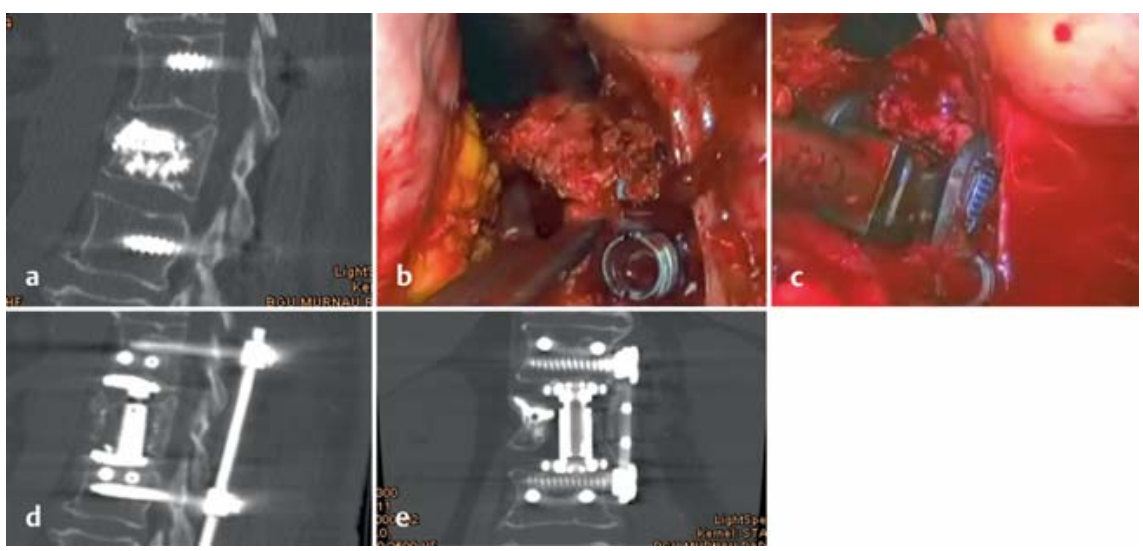

Abb. 6 a bis e Fehlgeschlagene Kyphoplastie bei 60-jähriger Patientin mit fortgesetzter massiver Beschwerdesymptomatik. Der Zement perforiert in die angrenzenden Bandscheiben, die Korrektur kann trotz additiver Instrumentierung nicht gehalten werden (a). Ventrale Korrektur thorakoskopisch gestützt zeigt das Bergen des Zements (b) und das Einbringen des Synex2-Cages (c). Postoperative CT-Kontrolle sagittal (d) und axial (e) zeigen die korrekte Stellung.

Bei fehlgeschlagener Kyphoplastie mit dann häufig in die Wirbelkörperzwischenräume dislozierten Zementanteilen müssen im Rahmen des ventralen Eingriffs der Zement geborgen und die angrenzenden Bandscheiben reseziert werden. Die weitere Vorgehensweise entspricht der oben beschriebenen, klassischen ventralen bisegmentalen Spondylodese mit expandierbarem Cage und winkelstabiler Platte (Abb. 6).

\section{Fazit}

Die Behandlung von Wirbelsäulenverletzungen muss zum Ziel haben, das Alignment des Achsenskeletts wiederherzustellen und insbesondere eine belastungsstabile vordere Säule zu rekonstruieren. Der dorsale Zugang dient dabei nach wie vor in der Akutphase als zuverlässiges Mittel der Reposition und Stabilisierung. Als isoliertes Verfahren allerdings hat er an Bedeutung verloren. Die differenziert eingesetzte ventrale Spondylodese - ob mono- oder bisegmental - unter Einsatz minimalinvasiver Verfahren vermag durch ihre minimierte Zugangsmorbidität $\mathrm{zu}$ überzeugen und wird auch dem älteren, aber biologisch jung gebliebenen Patienten angeboten. Zementaugmentierte Techniken gewinnen an Bedeutung, das adäquate Trauma beim älteren Patienten ist von der typischen osteoporotischen Fraktur nach Bagatelltrauma abzugrenzen. Auch rekonstruierende Korrektur- und Revisionseingriffe nehmen an Zahl und Bedeutung zu; gerade dieses Kollektiv aber sollte den Wirbelsäulenzentren überlassen werden.

\section{Literatur}

${ }^{1}$ Beisse R, Muckley T, Schmidt MH et al. Surgical technique and results of endoscopic anterior spinal canal decompression. J Neurosurg Spine 2005; 2: 128-136

2 Briem D, Windolf J, Lehmann $W$ et al. Ergebnisse der anterioren Fusion und therapeutische Konsequenzen. Unfallchirurg 2004; 107 : 1152-1161

${ }^{3}$ Bühren $V$. Verletzungen der Brust- und Lendenwirbelsäule. Unfallchirurg 2003; 106 : 55-68

${ }^{4}$ Disch AC, Knop C, Schaser KD et al. Angular stable anterior plating following thoracolumbar corporectomy reveals superior segmental stability compared to conventional polyaxial plate fixation. Spine 2008; 13: 1429-1437

5 Gonschorek O, Katscher S, Verheyden AP et al. Standardized $\mathrm{Ct} /$ fluoromatched navigation for the anterior stabilization of thoracolumbar spine fractures. In: Lemke HU, Inamura K, Doi K, Vannier MW, Farmann AG, eds. Computer assisted radiology and surgery. Philadelphia: Elsevier; 2005: 670-673

${ }^{6}$ Gonschorek O, Bühren V. Verletzungen der thorakolumbalen Wirbelsäule. Orthop Unfallchir up2date 2006: 1: 195-219

7 Gonschorek O, Merkel P, Maier M et al. Sportverletzungen an der Wirbelsäule. OP-Journal 2008; 24: 158-162

8 Josten C, Katscher S, Gonschorek O. Therapiekonzepte bei Frakturen des thorakolumbalen Überganges und der Lendenwirbelsäule. Orthopäde 2005; 34: 1021-1032

${ }^{9} \mathrm{Kim}$ DH, Jahng TA, Balabhadra RS et al. Thoracoscopic transdiaphragmatic approach to thoracolumbar junction fractures. Spine J 2004; 4: 317-328

${ }^{10}$ Knop C, Reinhold M, Röder C et al. Internet based multicenter study for thoracolumbar injuries: a new concept and preliminary results. Eur Spine J 2006; 15: 1687-1694

${ }^{11}$ Lange U, Edeling S, Knop C et al. Wirbelkörperersatz mit höhenvariablem Titan-Implantat. Unfallchirurg 2006; 109: 733-742

12 Magerl F, Aebi M, Gertzbein SD et al. A comprehensive classification of thoracic and lumbar injuries. Eur Spine J 1994; 4: 184-201

${ }^{13}$ Reinhold M, Schmölz W, Canto F et al. A new distractable implant for vertebral body replacement: biomechanical testing of four implants for the thoracolumbar spine. Arch Orthop Trauma Surg 2009; epub 4.2.2009

${ }^{14}$ Reinhold M, Knop C, Beisse R et al. Operative Behandlung traumatischer Frakturen der Brust- und Lendenwirbelsäule Teil I: Epidemiologie. Unfallchirurg 2009; 112: 33-45

15 Reinhold M, Knop C, Beisse R et al. Operative Behandlung traumatischer Frakturen der Brust- und Lendenwirbelsäule Teil II: Operation und radiologische Befunde. Unfallchirurg 2009; 112: 149-167

\section{Dr. med. Oliver Gonschorek \\ Leitender Arzt \\ Leiter der Wirbelsäulenchirurgie \\ Dr. med. Stefan Hauck}

Oberarzt der Wirbelsäulenchirurgie

Prof. Dr. med. Volker Bühren

Ärztlicher Direktor

Berufsgenossenschaftliche

Unfallklinik Murnau

Prof.-Küntscher-Straße 8

82418 Murnau

oliver.gonschorek@bgu-murnau.de 